\title{
Trivium
}

Revue franco-allemande de sciences humaines et sociales - Deutsch-französische Zeitschrift für Geistesund Sozialwissenschaften

5 | 2010

Sociologie des conventions

\section{Konventionen als Stützen der Handlung. Elemente der soziologischen Pragmatik}

\section{Nicolas Dodier}

Traducteur : Jörg Ohnacker

\section{CpenEdition}

Journals

Édition électronique

URL : http://journals.openedition.org/trivium/3565

DOI : 10.4000 /trivium.3565

ISSN : 1963-1820

Éditeur

Les éditions de la Maison des sciences de l'Homme

Référence électronique

Nicolas Dodier, « Konventionen als Stützen der Handlung. Elemente der soziologischen Pragmatik », Trivium [Online], 5 | 2010, online erschienen am 10 Januar 2010, abgerufen am 08 September 2020. URL : http://journals.openedition.org/trivium/3565; DOI : https://doi.org/10.4000/trivium.3565

Ce document a été généré automatiquement le 8 septembre 2020

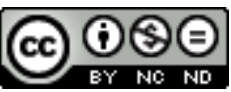

Les contenus des la revue Trivium sont mis à disposition selon les termes de la Licence Creative Commons Attribution - Pas d'Utilisation Commerciale - Pas de Modification 4.0 International. 


\title{
Konventionen als Stützen der Handlung. Elemente der soziologischen Pragmatik
}

\author{
Nicolas Dodier \\ Traduction : Jörg Ohnacker
}

\section{NOTE DE L'ÉDITEUR}

Wir danken Nicolas Dodier und der Zeitschrift Réseaux für die freundliche Genehmigung, diesen Artikel zu übersetzen.

1 In den Sozialwissenschaften lassen sich drei Herangehensweisen an die Problematik der Konventionen unterscheiden. Der erste Ansatz forscht nach allgemeingültigen Koordinationsmodellen, die davon ausgehen, dass allen Menschen bestimmte Kompetenzen gemeinsam sind. Für diesen Ansatz stehen gegenwärtig die Modelle von Habermas und Rawls ${ }^{1}$ sowie die neoklassischen Verfeinerungen des Marktmodells. Der zweite Ansatz will die Vielfalt an unterschiedlichen Typen von Gesellschaften und Gemeinschaften aufzeigen, die man für so deutlich voneinander geschieden hält, dass sich innerhalb einer jeden von ihnen homogene, den jeweiligen Mitgliedern gemeinsame Perspektiven ausmachen lassen. Im Gegensatz zum ersten Ansatz, der das Gewicht auf Modelle legt, die das menschliche Handeln in seiner Gesamtheit zu koordinieren vermögen, hebt der zweite die Existenz lokaler, räumlich oder zeitlich voneinander geschiedener Modelle hervor. Für den dritten Ansatz entstehen und existieren Konventionen in den beständig stattfindenden Abstimmungsprozessen zwischen den Akteuren im konkreten Handlungsvollzug. Er betont in erster Linie die pragmatischen Erfordernisse und Möglichkeiten der Realisierung augenblicksbezogener, kontingenten Umständen unterliegender Einigungen. Für diesen Ansatz steht in radikaler Form die von den Ethnomethodologen entwickelte Pragmatik wechselseitigen Verstehens (Garfinkel). ${ }^{2}$ 
2 Den vielversprechendsten Weg, der Frage nach den konventionenbasierten Grundlagen menschlichen Handelns nachzugehen, bietet zur Zeit sicherlich die Suche nach Querverbindungen zwischen diesen drei Ansätzen (die wir universalistisch, kulturell und pragmatisch nennen wollen). Die Arbeiten von Ricœur oder Walzer zeigen, dass es für die Beantwortung der allgemeinen Frage nach der Gerechtigkeit, die von Habermas und Rawls auf der Grundlage universalistischer Modelle abgehandelt wird, von größtem Interesse ist, die Existenz lokaler oder historischer Traditionen (Ricœur) ${ }^{3}$ bzw. die an verschiedenen Orten verankerten "Sphären der Gerechtigkeit« (Walzer) ${ }^{4} \mathrm{zu}$ berücksichtigen. Boltanski und Thévenot ${ }^{5}$ haben nachgewiesen, wie sich allgemeines Gerechtigkeitsempfinden in konkretes Handeln übersetzt und welche Konsequenzen sich auf pragmatischer Ebene aus der Existenz einer Pluralität kontrastierender Gerechtigkeitsmodelle ergeben. Die Vertreter des angelsächsischen Interaktionismus schließlich zeigen anhand zahlreicher empirischer Erhebungen, wie aus dem Handeln von unterschiedlichen Gruppen getragene "soziale Welten" hervorgehen (Strauss, Becker, Star und Griesemer, Fujimura). ${ }^{6}$ Sie stellen somit eine Verbindung aus dem kulturellen und dem pragmatischen Ansatz her.

3 Zur weiteren Vertiefung der zwischen diesen drei Ansätzen bestehenden Querverbindungen wird im Folgenden zunächst auf einen entschieden pragmatischen Ansatz zurückgegriffen, der auf die Beobachtung der Formen der Abstimmung von Personen untereinander oder mit ihrer Umgebung im konkreten Handeln ausgerichtet ist. Auf dieser Ebene werden die den Personen zur Verfügung stehenden konventionenbasierten Stützen untersucht, d.h. die Gesamtheit der Ressourcen, die zur Bildung einer - wenn auch nur minimalen - Gemeinsamkeit der Perspektiven beitragen, um Handlungen koordinieren zu können. Wie wir sehen werden, sind diese Stützen gleichermaßen in den Personen selbst und, in Gestalt von Objekten und Bezugspunkten, in externen Trägern verankert. Sie sind insoweit konventionenbasiert, als ihr Vorhandensein davon zeugt, dass ihnen Bemühungen vorausgingen, die Vorbedingungen für eine gemeinsame Orientierung der Personen untereinander oder zwischen den Personen und ihrem Umfeld zu schaffen. Es handelt sich folglich um die Ablagerungen einer Vergangenheit, die wie bei einem engen Verständnis des Begriffs der Konvention als eine explizite Übereinkunft erscheinen kann, ebenso jedoch als gegenüber anderen durchgesetzte Einzelinitiative, als stillschweigende Übereinkunft oder als fortschreitende Verankerung der Stützen durch Traditionen und Lernprozesse, für die sich schwerlich ein bestimmtes auslösendes Moment angeben lässt.

4 Im Weiteren werde ich die Hypothese entwickeln, der zufolge im Handlungsverlauf mehrere Koordinationsformen eine Verbindung miteinander eingehen. Ein zentrales Problem besteht an dieser Stelle darin, ein geeignetes Schema zu finden, mit dem sich diese dem Handeln inhärente Heterogenität untersuchen lässt. Für jeden der drei Ansätze steht jeweils etwas anderes auf dem Spiel: Hinsichtlich des pragmatischen Ansatzes geht es darum, von einem radikalen Pragmatismus ethnomethodologischer Prägung Abstand $\mathrm{zu}$ nehmen und somit die Erklärungskraft anderer, universalistischer oder lokaler Koordinationsmodelle aufzeigen zu können. Hinsichtlich des universalistischen Ansatzes sind solche Koordinationsmodelle, die bestrebt sind, unseren Erwartungshorizont zu erweitern, einer pragmatischen Prüfung zu unterziehen. Damit lässt sich ihre Verankerung in der Welt besser erfassen. Hinsichtlich des kulturellen Ansatzes soll die Hypothese eines engen Korrespondierens zwischen Koordinationsformen und Personengruppen relativiert und die Kombination dieser 
Formen im Ablauf der Handlungen ein und derselben Person untersucht werden. Mithilfe dieser Überlegungen lassen sich gewisse Orientierungen einer Pragmatik der Koordinationsformen genauer bestimmen.

\section{Allgemeiner Umriss einer soziologischen Pragmatik}

5 In der Soziologie wurde eine pragmatische Richtung oft mit einer fürchterlich restriktiven Fokussierung auf die unmittelbaren Bedingungen einer konkreten Handlung verwechselt. Soziologische Pragmatik lässt sich jedoch durch das Bestreben charakterisieren, hinsichtlich der Zukunft die größtmögliche Spannweite der Zeithorizonte der Akteure einzubeziehen, insofern diese im Handlungsablauf als eindeutig relevant erscheinen, und bezüglich der Vergangenheit eine Aufmerksamkeit für die gesamte Palette der im Gedächtnis gespeicherten Ressourcen zu entwickeln, die von den Akteuren tatsächlich mobilisiert werden. Im Folgenden sollen diese beiden Aspekte nacheinander untersucht werden.

6 Wie Schütz ${ }^{7}$ in seiner Phänomenologie des Handelns darlegt, sind die Zeithorizonte des Akteurs so miteinander verwoben, dass die Koordination mit dem Gegenüber sowohl von auf die unmittelbare Gegenwart bezogenen Sichtweisen als auch von in weitere zeitliche Ferne gerichteten Perspektiven abhängig ist. Zwischen den Zeithorizonten der Handlung besteht jedoch eine beträchtliche Asymmetrie: Die Akteure müssen sich stets mit der Gegenwart befassen, werden jedoch, je nach Situation, auch eine mehr oder weniger entfernte Zukunft in Betracht ziehen. Es mag deshalb seltsam erscheinen - es sei denn, man wollte gewagte Hypothesen über die menschliche Natur aufstellen -, wenn man, wie die strategischen Akteursmodelle, das Kalkül als vorrangig bei der Koordination des Handelns erachtet. Umgekehrt wäre es zweckmäßig, über eine Theorie des Handelns zu verfügen, die sich nicht von Anfang an durch eine grobschlächtige und wenig realistische strategische Mutmaßung selbst den Boden entzöge. Einer soziologischen Pragmatik, die auf die unterschiedlichen Formen des Engagements der Akteure in, des Sich-Einlassens auf ihr Handeln eingehen will, muss notwendigerweise eine Kritik an den Modellen vorausgehen, die das Kalkül als einzig mögliche Form der Beziehung berücksichtigen, die die Personen mit ihrer menschlichen und nichtmenschlichen Umgebung unterhalten. Anstatt die gegenwärtige Handlung auf im Hintergrund liegende hypothetische Motive zurückzuführen, empfiehlt es sich eher, zunächst die Interessen der Akteure als unmittelbares Handlungsziel in der Situation zu untersuchen. Im vorliegenden Ansatz sollen dementsprechend die kognitiven und moralischen Operationen des Umgangs mit der Umgebung (Bewertung, Identifikation, Beurteilung, Umgang mit Ungewissheit, Zurechtkommen mit den Kontingenzen des Augenblicks), sowie das Vorgehen bei der Koordination mit anderen im Handlungsverlauf offengelegt werden. Aus dieser weit gespannten theoretischen Konstellation heraus hat sich in der Soziologie an den Schnittstellen von Pragmatismus, Interaktionismus ${ }^{8}$, Ethnomethodologie, Ethnographie der Wissenschaften ${ }^{9}$ und den kognitivistischen Ansätzen des situierten Handelns ${ }^{10}$ ein pragmatischer Pol entwickelt.

7 Dieser Zugang zum Handeln schließt nicht aus, dass die Akteure möglicherweise bisweilen ein strategisches Verhältnis zum eigenen Handeln entwickeln können. Unter bestimmten Umständen betrachten sie, bewusst oder unbewusst, die Gegenwart auf der Grundlage eines bereits in eine nicht unmittelbar bevorstehende Zukunft gesetzten 
Ziels. Wenn die Annahme unrealistisch ist, dass die Akteure stets strategisch handeln, dann ist es ebenso wenig realistisch, grundsätzlich jegliche strategische Absicht im Handeln abzustreiten. Man sollte vielmehr, im Vergleich $\mathrm{zu}$ den strategischen Modellen, die Prioritäten umkehren. Handeln mit einer strategischen Ausrichtung enthebt die handelnde Person nicht der Auseinandersetzung mit den sich aus dem konkreten Handeln ergebenden Abstimmungsproblemen. Daraus resultiert die Notwendigkeit, die Akteure mit der Fähigkeit auszustatten, unmittelbar auf die Reize ihres Umfelds zu reagieren, was zur Folge haben kann, dass sie ihre Handlungspläne abändern (Interaktionismus) oder in bestimmten Fällen jeglichen Bezug auf einen Plan aufgeben müssen. ${ }^{11}$ Besonders aufschlussreich ist es zu zeigen, wie unterschiedliche, mehr oder weniger langfristige und mehr oder weniger flexible Formen der Vergegenwärtigung des Plans das konkrete Handeln orientieren..$^{12}$ Dabei kann die Figur der strategischen Verschleierung durchaus als spezifische Form der Beteiligung an der Handlung wieder auftauchen, um die Diskrepanzprobleme zu lösen, die sich aus den gleichzeitig an den Akteur gestellten Forderungen ergeben. ${ }^{13}$

Dieser Zugang zum Handeln setzt, zumindest in einem ersten Schritt, die Berücksichtigung der Phänomene voraus, die sich in einem relativ kurz bemessenen zeitlichen Rahmen abspielen. Rasch ablaufende Situationen, Szenen und Sequenzen werden zum vorherrschenden Element der Untersuchung, da sich ja die gesamte Handlung nicht direkt aus Plänen ableitet. Dieses Vorgehen erfordert eine gesteigerte Aufmerksamkeit für den unmittelbaren Augenblick und ein dementsprechendes methodisches Vorgehen (ethnographische Beobachtung, Aufzeichnung der Kurzsequenzen). Eine Analyse längerer Handlungssequenzen ist dabei nicht ausgeschlossen, allerdings hat sie nicht mehr den methodologischen Vorrang wie bei Vorgehensweisen, die von Anfang an auf der Grundlage eines strategischen Modells arbeiten.

Wenden wir uns nun der Vergangenheit der Handlung zu. Die Speicherelemente der konventionenbasierten Stützen der Handlung sind äußerst unterschiedlich geartet. Das können zunächst einmal interne, im Körper gespeicherte Stützen sein, die sich bewusst oder unbewusst mobilisieren lassen. Über Koordination können auch externe Stützen mobilisiert werden: Dabei handelt es sich um im Umfeld der Personen befindliche Entitäten. Diese »externen Gedächtnisse« weisen Elemente in Form von Spuren auf, die von den verschiedenen Protagonisten der Interaktion wiedererkannt werden können. Die Liste der konventionenbasierten Stützen erweist sich als ziemlich bunt zusammengewürfelter Haufen: Texte, Worte, Regeln, unterschiedliche Symbole, "kognitive Artefakte ${ }^{14}$ usw.

Der Abgleich der Handlungen zwischen Menschen und Objekten erfolgt über von den Menschen gespeicherte »Operationsketten«, »automatische Handlungsabläufe« ${ }^{15}$, aber auch über dazu passende, in den Objekten gelagerte "Anschlüsse « ${ }^{16}$, über in den Ablaufprogrammen eingeschriebene "Scripts" und technische Objekte, die das menschliche Verhalten antizipieren. ${ }^{17}$

11 Ziel einer soziologischen Pragmatik ist es, aufzuzeigen, wie diese unterschiedlichen Speicherelemente, ob sie nun extern oder intern, bewusst oder unbewusst sind, gleichzeitig im Handeln ihre Wirkung entfalten. Die gleichzeitig internen, unbewussten und relativ stabilen Träger haben die Aufmerksamkeit zahlreicher Ansätze auf sich gezogen, die sie als die Tiefenstrukturen des Handelns betrachtet haben. Man findet dieses epistemologische Primat der Tiefenstrukturen in der Geschichtswissenschaft 
(Primat der longue durée), der Anthropologie (Primat der Strukturen des Unbewussten) und der Soziologie (Primat der Dispositionen des Habitus). Diese Fokussierung hat dazu beigetragen, das Wissen über die eine der Koordinationsebenen wesentlich $\mathrm{zu}$ vertiefen. Ein Festhalten an einem solch einseitig ausgerichteten Zugang zum Handeln birgt jedoch die Gefahr, dass, angesichts der Verschiedenartigkeit der möglichen Formeln, die Fragen der Koordination auf ein kümmerliches Niveau reduziert werden. In einer solchen Perspektive ragt die Vergangenheit auf einem erstaunlich eingeschränkten Weg in die Gegenwart hinein: durch die Verinnerlichung mehr oder weniger bewusst übernommener Dispositionen in einem "psychischen Halbdunkel«. ${ }^{18}$ Dieses Primat kann dem pragmatischen Anspruch nicht genügen, die unterschiedlichen Wege, auf denen die Gegenwart von der Vergangenheit beeinflusst wird, in ihrer konkreten Ausgestaltung zu ermessen.

Weit davon entfernt, sich nur auf einen Teilbereich des Handelns zu konzentrieren, der sich lediglich auf die nahe Vergangenheit und die unmittelbare Zukunft beschränkt, sucht die pragmatische Richtung den Zugang zu den verschiedenen Modalitäten, mit denen die Personen zum jeweiligen Zeitpunkt eine Verbindung zwischen ihrer persönlichen Erfahrung, den vom Umfeld gelieferten Spuren der Vergangenheit und ihren Erwartungshorizonten herstellen. ${ }^{19}$ Kennzeichnend für eine soziologische Pragmatik ist die Öffnung hin zur beachtlichen Diversität der Formen des Verhältnisses zur Zeit ${ }^{20}$, die in den folgenden beiden Forderungen Ausdruck findet: Berücksichtigung der unmittelbaren Absichten des Handelnden als obligate Durchgangsstation für jegliches, auch auf weit entfernte Ziele gerichtetes Verhältnis zur Zeit; Analyse der vielfältigen Prozesse, über die die Vergangenheit in die Gegenwart einfließt. Aus diesem Anspruch einer soziologischen Pragmatik resultiert Kritik nach zwei Seiten hin: die Kritik am epistemologischen Primat des Kalküls und die Kritik am epistemologischen Primat der Tiefenstrukturen.

13 Man wird feststellen, dass diese Ausrichtung zwei Dinge impliziert. Zunächst kommt es $\mathrm{zu}$ einer Auflösung der kollektiven Akteure. Um beschreiben zu können, was von den Personen verlangt wird, wenn sie aufeinanderfolgende Szenen durchlaufen müssen, ist es aus methodologischer Sicht zweckmäßig, Einzelpersonen zu beobachten. Daher der Slogan »die Akteure verstehen«, womit weniger ein Nachvollziehen der Akteursperspektive gemeint ist, ebenso wenig eine Erklärung der Urteile, so als ob sich in ihnen die Wahrheit der Handlung zeigen würde, sondern das Auffinden von Methoden, mit denen sich die pragmatischen Zwänge nachvollziehen lassen, denen sie beim Handeln begegnen. Diese Strategie des Zugangs zum Handeln ist nicht gleichbedeutend mit einem Desinteresse für die aggregierten Entitäten. Sie impliziert jedoch, die (statistischen, monographischen, politischen ...) Aggregierungsvorgänge selbst zu untersuchen, die kollektiven Entitäten zu sprechen, zu denken, zu empfinden und zu entscheiden ermöglichen.

14 Die Soziologie desaggregierten Handelns erhebt zugleich den Anspruch, zu verstehen und zu vergleichen, wie sich die Konstruktion der aggregierten Entitäten vollzieht, und somit $\mathrm{zu}$ einer Soziologie der Aggregationsprozesse $\mathrm{zu}$ werden. Die zweite nennenswerte Richtungsänderung ist die Berücksichtigung der in der Situation vorhandenen Objekte. ${ }^{21}$ Das Interagieren mit der Umgebung beschränkt sich nicht auf Personen, sondern setzt auch eine Harmonisierung hic et nunc der Beziehungen zu den objekten voraus, die gleichzeitig wertvolle Stützen zur Verhaltenssteuerung abgeben, insbesondere in der heutigen, sich durch das Überhandnehmen technischer Objekte 
auszeichnenden Welt. Die Objekte liefern gemeinsame Stützen für die »Prüfungen«;22 sie können als Träger der externen Speicherung für die Bewältigung konkreter Situationen dienen und befreien die Akteure zum Teil von der Last des Sich-ErinnernMüssens: ${ }^{23}$ Sie bilden das Kontingent der "Aufschreiber «, denen die Menschen die Aufzeichnung dessen, was in der Welt vor sich geht, übertragen. ${ }^{24}$ Zur soziologischen Beschäftigung mit den Menschen kommt die Beschäftigung mit den Objekten hinzu. Die Begriffe »Kommunikation" bzw. "Interaktionen zwischen Menschen" erweisen sich nun als zu einengend für einen Zugang zum Handeln, der der Präsenz von Objekten Beachtung schenkt.

\section{Die Formen der Koordination des Handelns}

Der Begriff Koordination verweist auf das sehr allgemeine Bemühen um wechselseitige Handlungsabstimmung zwischen verschiedenen Protagonisten. Das besagt, dass die einzelnen Personen dem, was eine - flüchtige oder dauerhafte - Angleichung ihres Bezugs zur menschlichen oder nichtmenschlichen Umgebung darstellen kann, stark voneinander abweichende Bedeutungen zumessen. Die Umgangssprache wimmelt diesbezüglich von Termini, mit der sich die von den Personen beabsichtigte Art der Koordination und die damit einhergehenden Erwartungen genauer angeben lassen. Je nach Anlass ist die Rede von "Gerechtigkeit", "Effizienz«, „Vertrautheit«, »Vergnügen«, »Selbstverwirklichung«, »Liebe«, »Verschmelzung, »Können« usw. Wir stellen die Hypothese auf, dass jeder Harmonisierungsprozess seine Entsprechung in einer charakteristischen Gesamtheit konventionenbasierter Stützen und einer bestimmten Art und Weise hat, diese aufeinander abzustimmen. Die Koordination erweist sich nur dann als operativer Zugang, wenn angegeben werden kann, unter welchem Gesichtspunkt sie von den Personen angestrebt wird. Für jeden Koordinationstyp werden charakteristische Kombinationen interner und externer Stützen passend sein, die in der Form unterschiedlicher, bewusster und unbewusster Träger vorhanden sind. Die Phase der Inventarisierung und Parametrierung der konventionenbasierten Stützen ist nur eine Etappe einer pragmatischen Orientierung, die analysieren will, wie diese sich in der Handlung anordnen. Zur Untermauerung dieser Hypothese über die Koordinationsformen soll von zwei sich stark voneinander unterscheidenden Betrachtungsweisen der Koordination von Personen ausgegangen werden: den ethnomethodologischen Arbeiten zur Herstellung von Intersubjektivität, und dem Werk von Boltanski und Thévenot ${ }^{25}$ über gerechtfertigtes Handeln. Nachdem wir den Begriff der Koordination in mehrere Handlungsregimes unterteilt haben, können wir die sich zwischen diesen Regimes ergebenden Beziehungen untersuchen.

\section{Minimales wechselseitiges Verstehen}

16 Für Ethnomethodologen verweist die Koordination von Menschen auf eine grundlegende Eigenschaft des Handelns, nämlich seine »accountability«. ${ }^{26}$ Jede Person bringt, während sie handelt, gegenüber anderen zum Ausdruck, dass ihre Handlungen verständlich sind.

Dieses Angebot von Verständlichkeit wird vom Gegenüber aufgenommen, der es durch eigenes Handeln erwidert. Dieser Vorgang wird so fortgesetzt, dass jeder sich konkret am Verhalten des anderen ausrichtet und ihm gleichzeitig entsprechende Hinweise 
liefert, damit dieser sich wiederum daran ausrichten kann. Der gleichermaßen flüssige wie subtil organisierte Verlauf von Alltagsgesprächen liefert eindrückliche Beispiele für diese Koordinationsform. ${ }^{27}$ In der ethnomethodologischen Theorie ist Handeln immer doppeldeutig. Es ist der Umgebung zugewandt, um diese zu bewerten, zu kodieren, zu beurteilen oder sogar zu verändern. Über die Kontrolle der Zeichen von accountability ist es, in einer reflexiven Wendung, aber auch sich selbst zugewandt.

Für Ethnomethodologen gibt es zwei Typen konventionenbasierter Stützen des Handelns. Zunächst sind sie bei jeder Person in Gestalt von Fertigkeiten vorhanden und $\mathrm{ab}$ dem Zeitpunkt der Sprachbeherrschung in ihrem Handlungsprogramm eingeschrieben. Auf der externen Ebene treten die konventionenbasierten Stützen dagegen kaum zutage. Die tatsächlich aktiven Konventionen sind niemals vor der betreffenden Situation schon gegeben, sondern werden hic et nunc aus dem Verlauf des Interaktionsprozesses heraus generiert. Die Reflexivität jeder Handlung garantiert die Möglichkeit einer unmittelbaren Anpassung, die von der Person ad hoc »erfunden« und vorgenommen werden kann. Selbst wenn dieser Vorgang in eine Krise gerät, sind die Beteiligten zu Nachjustierungen in der Lage, die eine Fortsetzung koordinierten Handelns ermöglichen. Für die Ethnomethodologen spielt der Begriff der "ruhenden« Konvention keine Rolle bei der Handlungsanalyse: Keinerlei schriftliche oder mündliche Regeln, Worte, Vorgaben, Ratschläge kommen dem konventionenbasierten Grundelement gleich, das in den impliziten Prozessen besteht, in denen die pragmatische Bedeutung dieser Zeichen für die Durchführung einer koordinierten Handlung erkannt wird. Auf das Inventar dieser stillschweigenden Methoden und ihrer Voraussetzungen richtet sich der am konkreten Handeln orientierte Ansatz der Ethnomethodologen. Diese "Ethnomethoden« sind nicht mittels externer Träger in der Welt verankert. Sie sind nicht mit der Sprache identisch und sind auch nicht dementsprechend strukturiert, sondern bilden die Möglichkeitsbedingungen dafür, dass die Sprache die ihr eigene Funktion wechselseitiger Verständigung in der konkreten Situation erfüllen kann. Ebenso wie die Ethnographen bei ihrer Ankunft in einer Gesellschaft die ersten sind, die deren bis dahin nur in Form von Kompetenzen und allenfalls in mündlich überlieferten Orientierungen bestehenden Gebräuche in schriftliche Form übertragen, sind die Ethnomethodologen die ersten, die Ethnomethoden schriftlich niederlegen, was in der Regel durch die Auflistung charakteristischer Vorgänge geschieht. Um auf die entsprechenden Dimensionen der Koordination zugreifen zu können, müssen die Handlungen äußerst detailliert in Bild und Ton aufgezeichnet werden. Bislang erfolgte dies hauptsächlich anhand von Alltagsgesprächen ${ }^{28}$, sprachlichen oder gestischen Interaktionen in institutionellen Rahmen ${ }^{29}$, oder von Untersuchungen der Mensch-Maschine-Kommunikation. ${ }^{30}$

Im Hinblick auf die konventionenbasierten Träger des Handelns führen die Ethnomethodologen eine wichtige Dichotomie ein. Einerseits ziehen sie eine konventionenbasierte, allen gemeinsame Grundausstattung in Betracht, die mittels detaillierter empirischer Beobachtung konkreter Handlungen sichtbar gemacht werden kann, nämlich die Fertigkeiten als "Mitglied«, und die "Ethnomethoden« zur Herstellung der Intersubjektivität. Andererseits stellen sie fest, dass die Handelnden auf Regeln, Normen und Grundsätze Bezug nehmen. Diese letzteren, sozusagen allgemeinen Ressourcen werden ihrer Ansicht nach aus praktischen Gründen mobilisiert, die mit den Umständen der jeweiligen Situation zu tun haben. 

sich auch im Rahmen eines kognitiven und konversationellen engineering ${ }^{31}$ anwenden. Dabei stellt sich das Problem, dass die Analyse der Koordination auf ein Niveau des wechselseitigen Verstehens beschränkt wird, das man als minimalistisch bezeichnen könnte, wohingegen die höheren Ansprüche, die die Personen mit ihren reziproken Anpassungen verbinden können, unbeachtet bleiben. Um über diese minimale Ebene der Koordination hinauszugelangen, werde ich zunächst die Arbeit von Boltanski und Thévenot darstellen ${ }^{32}$, die in ihrer Untersuchung des an Gerechtigkeitserfordernissen orientierten Handelns die pragmatischen Bedingungen einer Koordination zu klären suchen, deren Anforderungsniveau für die Personen ungleich höher ist.

\section{Gerechtfertigtes Handeln}

Boltanski und Thévenot gehen von der Hypothese aus, dass es ein von allen gewöhnlichen Personen geteiltes Gerechtigkeitsempfinden gibt, zumindest in dem Maße, als sie als Zeitgenossen von der gleichen Tradition politischer Philosophie durchdrungen sind. In dieser von den Ethnomethodologen für umstandsabhängig erachteten Gesamtheit von Ressourcen (Regeln, Normen, Grundsätze, Gebräuche) suchen sie somit die beständigen und für die Rechtfertigung des Handelns verfügbaren konventionenbasierten Grundlagen. Dafür müssen sie die Personen, über die von den Ethnomethodologen vorausgesetzten Kompetenzen hinaus, mit zusätzlichen, ihnen allen gemeinsamen Fähigkeiten ausstatten. Sie gehen davon aus, dass jeder Mensch auf ein beschränktes Repertoire an Gerechtigkeitsmodellen Bezug nehmen kann. Der vorgeschlagene Rahmen umfasst insgesamt sechs "Gemeinwesen«: das familienweltliche Gemeinwesen, das industrielle Gemeinwesen, das Gemeinwesen der Inspiration, das marktwirtschaftliche Gemeinwesen, das Gemeinwesen der Meinung und das staatsbürgerliche Gemeinwesen. Die Verankerung dieser Gemeinwesen in der Welt wird unter anderem durch externe Entitäten stark unterschiedlicher Natur sichergestellt, die außerhalb der Personen als Stütze für das sich an Gerechtigkeitsmaßstäben orientierende Beurteilen dienen. Jedem dieser Gemeinwesen entspricht eine umfassende, mit einer ganzen Reihe von Wesen bevölkerte "Welt«: Objekte, mit Worten ausgedrückte Grundsätze, Prüfvorrichtungen, Beziehungen zwischen den Personen, zwischen den Personen und den Wesen und zwischen den 
externen Wesen selbst. All diese Wesen bilden zusammen die angemessene Umgebung für die innerhalb einer dieser Welten als gerecht geltende Koordination, während die Wesen der anderen Welten ignoriert oder angeprangert werden.

Damit diese Formen der Koordination Gestalt annehmen können, bedarf es einer umfassenden Grundausstattung an Konventionen, die sich als großes Reservoir individueller Fähigkeiten und externer Träger darstellt. Die Existenz dieses gemeinsamen Bestandes ist an den Umstand gebunden, dass wir in Gesellschaften leben, die auf politische, durch einen Traditionszusammenhang (im Sinne Gadamers) ${ }^{33}$ lebendig gehaltene Philosophien gegründet sind. Dieses gemeinsame Fundament ist nicht nur in die Personen eingeschrieben, sondern auch in Büchern, Institutionen, Dispositiven, Repräsentanten hinterlegt und wird ständig in den an Gerechtigkeit orientierten Disputen und der Konstruktion von "gerechtfertigten" Einigungen wiederaufgegriffen, weitergegeben und reaktiviert. Um die Verbindung zwischen den allgemeinen, aus dem Repertoire der gemeinsamen Welten geschöpften Ressourcen und den jeweiligen Einzelsituationen herzustellen, müssen die Personen von Mal zu Mal zahlreiche Vermittlungsakte bewerkstelligen. Im Gegensatz zu dem, was wir zur ethnomethodologischen Perspektive angemerkt hatten, können die Urteile hier jedoch eine mehr als ad hoc-Gültigkeit beanspruchen, insofern sie die von einem der Gerechtigkeitsmodelle definierten Erfordernisse berücksichtigen.

In Über die Rechtfertigung behandeln Boltanski und Thévenot somit das Problem der Konventionen bei konkreten Handlungen, indem sie es, wie die Ethnomethodologen, in zwei Bereiche aufteilen, deren Scheidelinie jedoch völlig anders verläuft. Auf der einen Seite geht es ihnen um das Vorhandensein einer gemeinsamen Basis von Konventionen in Form eines über externe Träger erweiterten und von Generation zu Generation weitergegebenen alltäglichen Gerechtigkeitssinns: die "gemeinsamen Welten«. Dieses Grundrepertoire an Konventionen bezieht sich auf die Sphäre gerechtfertigten Handelns. Das Streben nach Gerechtigkeit beschreibt schon per se eine Koordinationsform, die jedoch in sechs unterschiedlichen, jeweils einem der Gerechtigkeitsmodelle entsprechenden Formen umgesetzt werden kann. Dispute zwischen Personen sind das Resultat von Spannungen, die ihrerseits aus der Konfrontation zwischen den verschiedenen Gemeinwesen entstehen. Wenn sich diese Unstimmigkeiten in teilweise sehr heftigen Konflikten zwischen Personen niederschlagen können, dient dies gleichzeitig als Beleg, dass die Protagonisten über das gleiche Grundrepertoire verfügen, auch wenn jeder in der vorliegenden Situation sich jeweils auf anderes bezieht. Außerhalb dieses Bereichs gerechtfertigten Handelns sind die konventionenbasierten Grundlagen auf weniger allgemeine Geltung ausgerichtet. Sie können keinesfalls den Anforderungen an Allgemeingültigkeit genügen, wie sie für die an Gerechtigkeit orientierten Handlungen charakteristisch sind. In dieser terra incognita stellt sich die Frage der konventionenbasierten Grundlage des Handelns von neuem (Abbildung 2).

Abbildung 2: Ansatz des gerechtfertigten Handelns

\begin{tabular}{|c|c|}
\hline gerechtfertigte Handlungen & andere Handlungen \\
\hline $\begin{array}{c}\text { alltäglicher Gerechtigkeitssinn + gemeinsame } \\
\text { Welten }\end{array}$ & $\begin{array}{c}\text { (Anspruch auf geringere } \\
\text { Allgemeingültigkeit) }\end{array}$ \\
\hline
\end{tabular}




\section{Die Architektur der Koordinationsformen}

An diesem Punkt der Untersuchung können wir nun auf Grundlage der Arbeiten von Garfinkel sowie Boltanski und Thévenot zwei Formen von Koordination definieren. Die eine ist eine minimalistische Koordination zwischen den Handelnden, die in Gestalt eines fortgesetzten Vollzugs augenblicksbezogener wechselseitiger Verständigung erscheint und sich weder räumlich noch zeitlich verlagern lässt. Die andere ist dagegen eine maximalistische Koordination, die auf völlige Allgemeingültigkeit abzielende Urteile erfordert. Die zuerst beschriebene Koordinationsform kommt fast immer zur Realisierung: Sie ergibt sich sozusagen ohne das Wissen der Personen, insofern die dafür charakteristischen Abläufe im Vollzug der Tätigkeit von selbst vonstatten gehen. Ein Abgehen von dieser minimalen wechselseitigen Verständigung kommt einem Nachgeben gegenüber unkontrollierten Regungen gleich, wobei man keinerlei Bemühung mehr zeigt, das Verhalten des anderen zu interpretieren und ihm seinerseits ein verständliches Verhalten anzubieten. Man würde dabei in Tätigkeiten verfallen, denen keinerlei Konventionen mehr zugrunde liegen. Bei der ersten Form von Koordination erfolgte die Anpassung der Personen mehr oder weniger unbewusst, wohingegen der zweite Koordinationstyp Urteile, Überlegungen, Argumentieren und Selbstbesinnung in sprachlich-expliziter Form verlangt. Dieser Typus nimmt in einem eingeschränkten Kreis von Situationen Gestalt an: bei den gerechtfertigten Handlungen, in denen die Personen auf Gerechtigkeit bedacht sind.

Anhand dieser zwei Beispiele wird deutlich, in welch gegensätzlichen Formen die Koordination des Handelns in Erscheinung treten kann. Es bietet sich nun an, ihre Beziehungen genauer darzustellen und zu verdeutlichen, worauf dieser Begriff allgemein abzielt. Wir können somit eine allgemeine Architektur der Koordinationsformen skizzieren und die grundlegenden Hypothesen einer kombinierten Pragmatik der Handlungsregimes aufstellen, was entlang zweier Dimensionen erfolgen soll: 1. in der Zeit, wenn man den Personen bei ihren Tätigkeiten folgt; 2. im Raum, wenn sich das Interesse auf die Verteilung der Koordinationsformen richtet.

\section{Die Zeitlichkeit der Handlungsregimes}

29 Die Koordinationsformen stellen sich in zeitlicher Hinsicht auf dreierlei Weise dar: Gleichzeitigkeit, Aufeinanderfolge und Konfrontation. Erstere entspricht dem besonderen Status der minimalen wechselseitigen Verständigung. Solange man sich nicht in den Bereich der unkontrollierten Regungen begibt, ist wechselseitige Verständigung immer gegeben und führt zur Mobilisierung der von den Ethnomethodologen beschriebenen Kompetenzen und Operationen. Während sie sich untereinander verständigen, lassen sich die Personen jedoch zugleich auf verschiedene Koordinationsmodelle ein und spezifizieren die an ihr Handeln gestellten Anforderungen. Diese zusätzlichen Erwartungen können durch die Analyse der gerechtfertigten Handlungen gut veranschaulicht werden. Das Sich-Einlassen auf eine spezifische Koordinationsform ersetzt jedoch nicht den Prozess des Bemühens um minimale wechselseitige Verständigung, das weiterhin unablässig bestehen bleibt, sondern schafft zusätzlich neue Anforderungen. Somit besteht zwischen minimalem 
wechselseitigem Verständnis und allen anderen Koordinationsformen Gleichzeitigkeit (Abbildung 3).

Abbildung 3: Gleichzeitigkeit der Koordinationsformen

\begin{tabular}{|l|l|l|}
\hline \multicolumn{2}{|l|}{ minimale wechselseitige Verständigung } & $\begin{array}{l}\text { unkontrollierte } \\
\text { Regungen }\end{array}$ \\
\hline $\begin{array}{l}\text { sich an der Gerechtigkeit orientierende } \\
\text { Handlungen }\end{array}$ & $\begin{array}{l}\text { andere } \\
\text { Koordinationsformen }\end{array}$ & \\
\hline
\end{tabular}

31 Der Begriff der Koordinationsform führt zur Hypothese einer wechselseitigen Integration der unterschiedlichen Ressourcen. Dem Erfordernis der Koordination wird nur dann genüge getan, wenn die Personen auf eine bestimmte Art und Weise die aus einer charakteristischen Menge geschöpften Ressourcen miteinander verbinden. Die konventionenbasierten Bestandteile der Koordination sind in den einzelnen Regimes nicht etwa isoliert, sondern in Komplexen von Regeln, Worten, Objekten, das heißt in Dispositiven, zusammengefügt. Wird eine Regel, ein Wort oder ein Objekt von einer Person aufgegriffen, muss diese mit diesem Element zugleich alle ihm innerhalb eines Dispositivs assoziierten Elemente heranziehen. So setzt etwa der Arzt bei einer medizinischen Untersuchung mit dem Stethoskop einen festen Ablauf von klinischen Handlungsschritten in Gang, in dessen Rahmen die Geräusche, die er mittels seines Instruments hört, eine diagnostische Bedeutung erhalten. ${ }^{34}$ Der technische Gegenstand erfüllt seine Funktion in einer Handlung nur dann, wenn alle Verbindungen geknüpft sind, die seine Verortung in einem Netzwerk gewährleisten..$^{35}$ Das einzelne Wort wird nur über das Sprachspiel, das es in das Gewebe der Alltagssprache einbindet, in eine konkrete Situation eingebettet. ${ }^{36}$ Ressourcen stehen somit nicht isoliert, sondern sind in Komplexen miteinander verbunden. Will man ein Element herausgreifen, kommt das gesamte Geflecht, in das es eingebettet ist, zum Vorschein.

Dieser Prozess wird im Begriff des Engagements bzw. des Sich-Einlassens oder Involviertseins der Personen deutlich: Wenn sie einmal dabei sind, in bestimmter Hinsicht die Koordination mit einem Gegenüber herstellen zu wollen, müssen sie sich gezwungenermaßen auf die Gesamtheit der bereits von der Vergangenheit bereitgestellten Verbindungen einlassen. Sie haben nicht nur das Stethoskop als technisches Objekt oder das einzelne Wort vor sich, sondern die gesamte medizinische Untersuchung, ein soziotechnisches Netzwerk oder ein Sprachspiel. Mit der Annahme von Koordinationsregimes soll die grundlegende Eigenschaft des Handelns betont werden: seine Rigidität. Die konventionenbasierten Stützen bilden untereinander Komplexe. Solche Verbindungen sind zwar nicht vollkommen irreversibel, aber es ist bisweilen ein beträchtlicher Aufwand nötig, um das wieder voneinander zu trennen, was die Vergangenheit, ohne dass dies beabsichtigt war, verbunden hat. Die Anbahnung dieser Verbindungen ebnet den Weg, den unsere Handlungen nehmen.

An dieser Stelle muss darauf hingewiesen werden, dass die wechselseitige Anpassung der Handlungen nicht grundsätzlich ein gleich starkes Sich-Einlassen beider Seiten auf ein und dieselbe Koordinationsform voraussetzt. Wie bereits bemerkt, ist das minimale wechselseitige Verständnis ständig gegenwärtig, um eine Anpassung zwischen Personen zu erreichen, wobei es keine Rolle spielt, in welche Formen der Koordination 
die Personen sonst noch involviert sind. Um diesen möglichen Mangel an Symmetrie zu vermeiden, behaupten wir, dass die Personen jeweils in unterschiedliche Handlungsregimes involviert sind. Sie können ihre jeweiligen Handlungen koordinieren, ohne in dasselbe Regime involviert zu sein. Ein gleichzeitiges Involviertsein in der gleichen Koordinationsform, über ein minimales gegenseitiges Verständnis hinaus, stellt somit lediglich einen besonderen Fall von Koordination dar.

Zwischen den einzelnen Regimes variiert das Integrationsniveau der Ressourcen beträchtlich. In der Untersuchung von Boltanski und Thévenot verfügt jedes Regime über eine starke Integrationskraft. Dabei wirkt sich der Umstand, dass diese Handlungen auf Gerechtigkeit abzielen, auf die Erscheinungsform der Regimes aus: Jede gemeinsame Welt, die ein gerechtes Gemeinwesen mit Anspruch auf Allgemeingültigkeit verkörpern will, muss eine Gesamtheit mit einem immensen Spektrum an Wesen sein. Das Integrationsniveau wirkt sich auf die sich zwischen den unterschiedlichen Regimes bildenden Beziehungen aus. Da jede "gemeinsame Welt" vorgibt, für sich allein ein Gemeinwesen umzusetzen, befindet sie sich zwangsläufig im Widerspruch zu jeder anderen "gemeinsamen Welt«. Jedes Regime bildet eine »Welt« im eigentlichen Sinn des Wortes, das heißt, es verbindet Wesen zu einer umfassenden, sich selbst genügenden und exklusiven Gesamtheit.

In seinem Buch Rahmenanalyse ${ }^{37}$ behandelt Goffman die Koordination von Handlungen auf wesentlich niedrigeren Niveaus der Integration. Man kann unschlüssig sein, ob man die fast unmerklichen Haltungsveränderungen, durch die sich bestimmte Erfahrungsrahmen auszeichnen, tatsächlich als »Handlungsregimes" oder "Koordinationsform" bezeichnen will. Die Untersuchung des Involviertseins des Einzelnen erfolgt hier in erster Linie auf der Grundlage seiner subjektiven Erfahrung. Die Koordination mit dem anderen wird über Gesten, Objekte und Verhaltensweisen, vor allem jedoch über innere Verschiebungen in der Art der Sicht der Dinge sichergestellt. Goffman widmet sich der Erstellung eines umfangreichen Inventars möglicher Rahmen und illustriert diese durch Unmengen von Beispielen. Er verweist auf die unzähligen Möglichkeiten im Umgang mit den Anordnungen und die unendliche Zahl der Rahmenbrüche, etwa in Gestalt von Verwirrung, Verlegenheit, Ungewissheit oder Unschlüssigkeit. Die Rahmen können miteinander harmonieren oder in Widerspruch zueinander geraten. Diese Rahmenarchitektur nimmt sowohl mit zunehmender innerer Erfahrung als auch in den Interaktionssequenzen mit anderen Wesen Gestalt an. Bei Goffman stellt die Verbindung einiger weniger Ressourcen einen Rahmen dar, wobei die Kombinationsmöglichkeiten zwischen den Rahmen sehr offen bleiben. Seine Form der Klassifikation und sein Vorgehen erinnern eher an die Pragmatik Austins ${ }^{38}$ als an die von Boltanski und Thévenot vorgenommene axiomatisierte Anordnung der Handlungsregimes in Welten.

Zwischen diesen beiden Extremen gibt es jedoch auch Handlungsregimes, die über die Rahmen der Erfahrung hinausgehen und mehr Verbindungen zu externen Trägern aufweisen, aber weniger integriert und dramatisch gespannt sind als die an Gerechtigkeit orientierten Handlungsregimes. In einigen neueren Arbeiten wird gezeigt, dass in der gegenwärtigen Medizin unterschiedliche Handlungsregimes nebeneinander bestehen: Es werden etwa »Formate« der Arzt-Patienten-Beziehung anhand einer Konversationsanalyse von Konsultationsgesprächen aufgezeigt ${ }^{39}$, verschiedene »Formen der Entschlüsselung von Schmerzen« anhand eines minutiös durchgeführten Vergleichs von Sprechstundenverläufen in Spezialkliniken 
unterschieden ${ }^{40}$ sowie unterschiedliche Formen der "Passung der Individuen« bei medizinischen Gutachten anhand von Fällen aus der Arbeitsmedizin identifiziert. ${ }^{41}$

Diese Hypothese der wechselseitigen Einbeziehung der Ressourcen innerhalb der Koordinationsformen ist die Grundlage des hier entwickelten Modells des Handelns und hat erhebliche Auswirkungen auf die Zeitlichkeit des Handelns. Um auszudrücken, dass die Personen eine Gesamtheit von untereinander verbundenen Ressourcen mobilisieren müssen, sagen wir, dass diese Personen in einem Regime »engagiert« bzw. in dieses »involviert« sind. Der Begriff des Regimes unterstellt dem Handeln eine gewisse Rigidität. Folgt man jedoch den Personen in ihren konkreten Handlungen, stellt man fest, dass sie selten im selben Zustand verharren. Im Allgemeinen durchlaufen die Personen eine Reihe aufeinanderfolgender Szenen, in denen sie das Regime wechseln: Dies geschieht entweder unter dem Druck der angetroffenen Dispositive oder anderer Personen, schließlich auch in Abhängigkeit von ihren inneren Einstellungen. In diesen Übergängen zwischen Regimes zeigt sich die in der gegenwärtigen Welt dem Handeln innewohnende Heterogenität.

Diese Heterogenität ergibt sich in erster Linie aus der Pluralität der von einem jeden beherrschten Koordinationsformen. Im Gegensatz $z u$ den $z u$ einfach gestrickten Hypothesen der kulturellen Ansätze verfügen Personen über die Möglichkeit und Fähigkeit, sich auf mehrere Handlungsformen einzulassen. Jeder lagert im Verlauf seines Werdegangs in sich ein buntes Gemisch von Handlungspotentialen ab. Die Formen des Handelns stellen sich uns als eine Palette an Möglichkeiten dar, die von keiner Instanz zusammengeführt werden können. Angesichts einer konkreten Situation steht jeder an einem Kreuzweg, der mehrere Formen offenhält, sich auf eine Handlung einzulassen. So lässt sich etwa ein Problem der Gerechtigkeit in einer industriellen, marktwirtschaftlichen oder staatsbürgerlichen Form angehen. Man kann sogar unschlüssig sein, ob ein Problem unter dem Aspekt der Gerechtigkeit oder der Liebe $\mathrm{zu}$ betrachten ist. $^{42}$ Wir haben bereits darauf verwiesen, dass diese Handlungsregimes nicht ausschließlich in Personen, sondern auch in externen Stützen verankert sind. Diese Annahme einer Trennung von Handlungsregimes und Personen hat hier weitreichende Folgen. In zahlreichen Situationen müssen die Personen ihr eigenes Sich-Einlassen mit den Stützen konfrontieren, auf die sie außerhalb ihrer selbst stoßen. Ihr Sich-Einlassen folgt nicht ausschließlich einem inneren Hin und Her zwischen Zuständen und Dispositionen, sondern wird, bisweilen ihren ersten Regungen zuwiderlaufend, von den externen konventionenbasierten Stützen gesteuert. Im äußersten Fall veranlasst sie der Druck der Situation, von einem Regime in ein anderes »umzuschwenken«.

Dementsprechend ist die innere Heterogenität des Handelns die Regel, seine Einheitlichkeit die Ausnahme. Tatsächlich bedarf es äußerst wirksamer Verfahren zur Vereinheitlichung der möglichen Bezugnahmen, um die Personen auf eine einzige Form des Handelns auszurichten. Eine andere Möglichkeit wäre, dass die Person sich unerschütterlich danach ausrichtet, sich in Einklang mit ihrer Umgebung zu bringen, und dies in so umfassender Weise, dass dabei alle Unwägbarkeiten der Existenz einbezogen werden können. Mit Sicherheit ermöglichen bestimmte auf Harmonie bedachte religiöse Lebensformen ein sozusagen im Fluss befindliches Dasein, bei dem, wie in der Studie Leenhardts ${ }^{43}$ zur religiösen Phänomenologie dargestellt, die Personen in die Zeitlichkeit eines geeinten Universums eingetaucht sind. 

Koordinationsformen. Man kann nun einheitliche, von einem Regime beherrschte Zeitsequenzen isolieren und die Momente identifizieren, in denen es $\mathrm{zu}$ Diskontinuitäten kommt, die sich durch den Wechsel der Personen von einem Regime in ein anderes ergeben. Die Koordinationsformen treten jedoch auch in ein Verhältnis der Konfrontation zueinander: wenn mehrere, in unterschiedliche Regimes involvierte Personen aufeinandertreffen, oder die Situationen für andere Regimes als diejenigen eingerichtet sind, auf die sich Personen weiter einlassen wollen, oder im Zuge eines Zusammenstoßes mit nicht-menschlichen, für andere Formen des Handelns präparierte Entitäten. ${ }^{44}$

Die Untersuchung dieser Konfrontationen und der sich daraus ergebenden Kombinationen stellt einen der zentralen Bereiche einer soziologischen Pragmatik dar. Auch hier wirkt sich das Niveau der Integration der Ressourcen auf die Ausgestaltung der Kombinationen aus. Sind die Ressourcen in "Welten« integriert, die, wie im Fall des an Gerechtigkeit orientierten Handelns, keine Parasiten zulassen, entwickeln sich die Konfrontationen $\mathrm{zu}$ "spannungsgeladenen " Situationen ${ }^{45}$, und die Kombinationen werden $\mathrm{zu}$ unbeständigen Kompromissen. ${ }^{46}$ Bei einer geringeren Integration der Ressourcen können sich aus der Konfrontation Gebilde ergeben, die für weitere Umgestaltungen offen sind. ${ }^{47}$ Bestimmte Koordinationsformen, etwa diejenigen, die mit der Dynamik soziotechnischer Netzwerke ${ }^{48}$ zusammenhängen, zeichnen sich sogar durch den hartnäckigen Versuch aus, heterogene Elemente zu neuartigen Hybriden miteinander zu verbinden, die man dann ständig zu stabilisieren und in Umlauf $\mathrm{zu}$ bringen versucht.

em Begriff der Koordinationsform soll somit auf zwei Eigenschaften des Handelns verwiesen werden: nämlich Rigidität, die sich durch die Diskontinuitäten in der Abfolge der von den Personen durchlaufenen Szenen in Gestalt aufeinander folgender Engagements, Akte des Sich-Einlassens, äußert, und Komplexität $t^{49}$, die sich aus der Pluralität der nebeneinander bestehenden Regimes ergibt und eine Quelle von Spannungen und Kombinationen darstellt. Knüpft man an den Koordinationsbegriff zu niedrige Anforderungen, unterschätzt man die Rigidität des Handelns. Dies ist bei den Ethnomethodologen der Fall, die sich auf die Untersuchung eines minimalen wechselseitigen Verständnisses beschränken. Allgemein gesagt, neigt eine Pragmatik der Konventionen, die der inneren Solidität der Dispositive nicht Rechnung trägt, zur Überschätzung des fließenden Charakters des Handelns. Zudem geht es an der Komplexität des Handelns vorbei, wenn man die Handlungsregimes zu stark an die Personen bindet und so die Übergänge von einem Regime zum anderen nicht erfasst. Hierin liegt die Schwäche der kulturellen Ansätze.

In der von uns aufgezeigten Perspektive wird die soziologische Pragmatik zu einer »kombinatorischen" Pragmatik, die sich mit den Kombinationsmöglichkeiten der verschiedenen Regimes beschäftigt. Sie orientiert sich an einer phänomenologischen Soziologie, wenn Handlungen derselben Person analysiert und in aufeinanderfolgenden Zeitpunkten beobachtet werden, und sie gibt Aufschluss über die Operationen, mit denen eine Person die Heterogenität des Handelns angeht: so zum Beispiel die Errichtung einer Fassade und eines inneren Raums ${ }^{50}$, die Aufspaltung des Selbst in mehrere Persönlichkeiten, die Wahrung der Kontinuität und letztlich der Identität. ${ }^{51}$ Diese Pragmatik beschäftigt sich auch mit den Prozessen, in deren Verlauf die aufeinanderfolgenden Szenen zu einem Gesamtkomplex zusammengefügt werden, wie 
sprachliche Doppel- oder Mehrdeutigkeiten sowie das Vornehmen von Übersetzungen. Die Koordination wird somit aus der privilegierten Perspektive des Handelnden gesehen, dessen Handeln über verschiedene Sequenzen hinweg erfasst wird; dabei wird deutlich, was er alles tun muss, um die diskontinuierlich aufeinanderfolgenden Momente $\mathrm{zu}$ einem Ganzen $\mathrm{zu}$ arrangieren. Die soziologische Pragmatik wird zur Soziologie der Interaktion, wenn sie eher die verschiedenen Teilnehmer einer Zusammenkunft zusammen in den Blick nimmt als ihrem Tun jeweils einzeln nachzugehen. Man kann beispielsweise die Dynamiken des Disputs ${ }^{52}$ und die zur Herstellung von Einvernehmen verwendeten Figuren (Kompromiss, Vergessen, Vergeben ...) aufzeigen.$^{53}$ Bezieht sich die Untersuchung auf die Erfindung neuer, aus der Konfrontation zwischen Regimes entstehender Arrangements ${ }^{54}$, läuft das Vorgehen eher auf eine Soziologie der Montage hinaus. Die Beobachtung der ruhenden Dispositive hat dann Vorrang vor der Beobachtung der Handlungssequenzen. Dieser Ansatz richtet sich auf längere, bisweilen sogar historische Zeiträume, insbesondere in den Untersuchungen über wissenschaftliche und technische Neuerungen..$^{55}$

\section{Die Verteilung der Handlungsregimes im Raum}

Zur Vervollständigung der Architektur der Koordinationsformen bedarf es noch der genaueren Darstellung ihrer Organisation im Raum. Wie bereits ausgeführt, sind diese Formen auf zweierlei Weise simultan in der Welt verankert: einmal »intern« über die Personen und ihre Fähigkeiten, sich zu engagieren bzw. einzulassen, und einmal »extern« mittels nicht-menschlicher Entitäten wie Dingen, Tieren, Objekten, Worten, Texten usw. Manche Formen schlagen, um sich entfalten zu können, ganz bestimmte Wege ein. Die Stützen des Regimes der Agape etwa sind ihrer Natur nach in den Personen verortet. ${ }^{56}$ Selbst der Rückgriff auf Sprache wird problematisch. Wir haben zudem festgestellt, dass die minimale wechselseitige Verständigung der Ethnomethodologen auf internen Kompetenzen und nicht auf materiell gestützten Vorgehensweisen basiert. Dagegen kann sich die Dynamik der soziotechnischen Netzwerke nur bei starker Zunahme der technischen Objekte und Hybride jeglicher Art entfalten, die dann ihrerseits als Stützen zur Erweiterung der Netzwerke beitragen. ${ }^{57}$

Wir wollen zunächst die Verankerung mittels nicht-menschlicher Entitäten betrachten. Jede externe Entität birgt, für sich allein genommen, in der Regel eine beträchtliche Unbestimmtheit, $\mathrm{zu}$ welcher Form von Koordination sie die Personen veranlassen könnte. Man kann sagen, dass sie bezüglich der Koordinationsformen, mit denen sie assoziiert werden kann, »neutral« ist. Es ist nicht möglich, zwischen dieser Entität (Tier, Objekt, Regel, Wort ...) und einem Handlungsregime eine genaue Entsprechung herzustellen. Eine Holzbank, für sich allein genommen, ist vollkommen neutral. Um ihre spezifischen Eigenschaften hervorzuheben, müsste man sie in einen Gesamtzusammenhang stellen, der die möglichen Formen des Engagements der Person beschränkt. Als Teil eines Ganzen, dessen Ausrichtung aus sich heraus deutlich wird, tritt die Bank aus ihrer Neutralität heraus und erweist sich als wahrhaft spezifisch für eine bestimmte Form der Koordination. Dieselbe Bank steht jetzt im Parlament und ist mit einer großen Zahl anderer Dispositive verknüpft. Die darauf sitzenden Personen engagieren sich in einer Form staatsbürgerlicher Koordination und sind bereit, die entsprechende Sprache, die entsprechenden Stimmung und weitere Objekte einzusetzen. Ein weiteres Beispiel ist das Stethoskop: Es steht in Verbindung zu der Liege, auf die sich der Patient legen soll, und zu anderen Untersuchungsinstrumenten 
(Blutdruckmesser, EKG ...), die ihrerseits mit anderen Medizintechnologien, anderen Orten oder Texten (Gebrauchsanleitungen, nosographischen Tabellen, Behandlungskatalog) und einer Patientenakte in Verbindung stehen; in ihrer Gesamtheit richten sie das Engagement des Arztes darauf aus, in Bezug auf das Individuum eine klinische Einstellung einzunehmen. Andere Entitäten sind liminale Entitäten $^{58}$, die weiten Spielraum für eine Verwendung in mehreren simultanen Koordinationsformen lassen. Sie können zum Beispiel aus dem Bemühen um einen Kompromiss zwischen mehreren Formen des Handelns angefertigt worden sein und noch die Spuren dieser Zusammensetzung tragen. Dies gilt etwa für einen statistischen Fragebogen, der die Anforderungen verschiedener Akteure (Statistiker, Ärzte, Ministerialbeamte, Epidemiologen) berücksichtigt, durch deren Hände er nacheinander gehen wird. ${ }^{59}$ Sie können jedoch auch für eine bestimmte Form des Handelns geschaffen worden sein oder deren Spuren tragen, und dann in einem anderen Regime Verwendung finden, ohne dass dies der Absicht desjenigen entspricht, der sie entworfen hat. Das Basteln ist ja die Kunst, in Objekten Verwendungsmöglichkeiten zu entdecken, für die sie ursprünglich nicht konzipiert waren. Die externen Entitäten richten somit mittels flexibler Führungsmechanismen die Personen auf bestimmte Formen der Koordination aus. Je nach Fall sind sie weitgehend unbestimmt (neutrale Entität), können sich simultan auf mehrere Formen hin orientieren (liminale Entität), oder sind, restriktiver, an eine einzige Form gebunden (spezifische Entität).

Die Verteilung der nicht-menschlichen Entitäten steuert somit die räumliche Verteilung der Regimes des Handelns. Die Koordinationsformen sind in der Umgebung bereits mehr oder weniger vorhanden, bevor die Personen die Szene betreten. Sie lassen sich kartographisch verzeichnen. Man kann eine Soziologie der Verteilung der Formen des Handelns betreiben (nach Land, nach Unternehmen ...) und dabei, am Ende eines weiten Umwegs, wieder auf strukturelle Themen stoßen. Bessy hat zum Beispiel Übereinstimmungen zwischen Unternehmensmodellen und Wirtschaftszweigen festgestellt. ${ }^{60}$ Verglichen mit den klassischen strukturellen Ansätzen bietet dieses Verfahren den Vorteil, dass man die Verteilung der Handlungsregimes ausmessen kann, ohne dabei die Dynamik des Handelns außer Acht zu lassen. Im Übrigen hat Mol ${ }^{61}$ gezeigt, dass sich in der Art und Weise, wie die Entitäten untereinander verknüpft sind, unterschiedliche "Topographien" abzeichnen. Mol verwendet das Beispiel der "Anämie« in der modernen Medizin: Für diesen Begriff koexistieren mehrere Definitionen (physiologisch, klinisch, labortechnisch) nebeneinander. Wenn man nun entsprechend einem "topographischen « Ansatz eine Karte der Verwendung dieser Definitionen erstellt, stellt man fest, dass die Konstellationen der Koexistenz ihrerseits unterschiedlich ausfallen: Konflikte, zugelassene und friedliche Pluralität, Verschränkung der unterschiedlichen Kenntnisse, wenn das Blut zwischen Krankenhaus, Labor und Arztpraxis zirkuliert. Man muss sich daher von der Vorstellung eines in "Regionen" unterteilten Raums lösen und andere Formen der Verankerung ins Auge fassen. ${ }^{62}$

47 Als nächsten Schritt wollen wir die Verankerung der Handlungsregimes in den Menschen näher betrachten. Wie bereits gesagt, verfügt in unserem Modell jede Person über mindestens zwei Arten von Kompetenzen: die Fähigkeit, sich auf Koordinationsformen einzulassen, und die Fähigkeit, den Übergang von einer Form zur anderen $\mathrm{zu}$ bewerkstelligen. Bestimmte Handlungsregimes sind in jedem von uns verankert, und die Beherrschung dieser Koordinationsformen ist Teil der Grundausstattung jedes normalen Menschen. Die Fähigkeit zu minimalem 
wechselseitigem Verstehen ist eine dieser Formen, ein alltäglicher Gerechtigkeitssinn eine weitere. Ihre Untersuchung fällt in den Bereich einer Soziologie des common sense. Unter diesem Blickwinkel untersucht Boltanski ${ }^{63}$ die pragmatischen Bedingungen des Regimes der Agape, und Thévenot ${ }^{64}$ zeigt auf, dass es verschiedene Niveaus hinsichtlich dessen gibt, was jeweils eine angemessene Handlung ist. Da die Personen von einer Form in eine andere wechseln können, werden diese Koordinationsformen nicht ständig von ihnen aktualisiert; sie tragen jedoch, als Potentialitäten, beständig die Möglichkeit in sich, sich auf eine von ihnen einzulassen, wenn die jeweiligen Umstände dafür entsprechende Anhaltspunkte bieten. Betrachtet man die in den Bereich des common sense fallenden Formen des Handelns, kann es zwar hinsichtlich der Performanz Unterschiede zwischen den Personen geben (insbesondere in Abhängigkeit der von ihnen vorgefundenen externen Stützen, die sie in dieses oder jenes Handlungsregime lenken), hinsichtlich der Fähigkeiten gibt es jedoch keinen Unterschied; hier ist eher der Begriff "Kompetenzen« zutreffend, im Sinn eines Gepäckstücks, das jeder mit sich trägt.

Im Gegensatz dazu gibt es Formen der Koordination, die nur von fest umrissenen Personengruppen beherrscht werden. Diese Regimes können nur bei Personen mit einer entsprechenden Ausbildung Gestalt annehmen. Sie werden weder über die Umgangssprache noch über einen uns allen gemeinsamen Überlieferungszusammenhang vermittelt, sondern über spezifische Unterweisungen oder Erfahrungen. Bei diesen Regimes bestehen Unterschiede sowohl hinsichtlich der Performanz als auch hinsichtlich der Fähigkeiten. Ihre Untersuchung fällt in den Bereich einer Soziologie der lokalen Formen von Koordination. Man kann zum Beispiel feststellen, dass bestimmte Handlungsregimes dem Erwerb spezieller Kenntnisse und Fähigkeiten entsprechen, die auf bestimmte Orte beschränkt und bestimmten Personengruppen vorbehalten sind. Personen, die diese speziellen Kenntnisse und Fähigkeiten nicht erworben haben, sind nicht fähig oder verstehen sich nicht darauf, sich auf diese Formen des Handelns einzulassen, oder sie stehen ihnen schlicht teilnahmslos gegenüber. Sich mit anderen in diesen spezialisierten Bereichen koordinieren zu wollen, wird von ihnen erst gar nicht in Betracht gezogen. So gibt es beispielsweise bestimmte Formen, Individuen zu betrachten, zu behandeln, auf ihre Klagen und Leiden zu reagieren, die für den Blick des Arztes charakteristisch sind (Abbildung 4). ${ }^{65}$

Abbildung 4: Verteilung der Koordinationsformen

\begin{tabular}{|c|c|}
\hline common sense & lokale Formen \\
\hline minimales wechselseitiges Verstehen & \\
gerechtfertigte Handlungen & spezialisierte Formen \\
Agape & (z.B. der ärztliche Blick) \\
die angemessene Handlung usw. & voneinander abgeschottete Territorien usw. \\
\hline
\end{tabular}

Die dem common sense zugehörigen Handlungsregimes sind heterogen, sind jedoch in Form von Kompetenzen bei jeder Person nebeneinander vorhanden. Betrachtet man lokale Formen, kann man auf Situationen stoßen, in denen ein Handlungsregime nur von einem Teil der an der Begegnung beteiligten Personen beherrscht wird. Solche 
Situationen schaffen neue Probleme der Abstimmung zwischen den Personen. Solange man sich im Bereich des common sense bewegt, haben die Personen einen symmetrischen Zugang zu den Formen der Koordination. Sie sind imstande, sie zu erkennen und sich, im gegebenen Fall, auf sie einzulassen. Wenn, im umgekehrten Fall, eine Person in einer lokalen Form engagiert ist, kann es möglich sein, dass manche Protagonisten nicht über die Fertigkeit verfügen, sich auf diese Form einzulassen. Das Engagement ist dann asymmetrisch. Dies führt aus der Sicht der Person, die diese Koordinationsform nicht beherrscht, zu einer beträchtlichen Unsicherheit bezüglich der Art der von ihr erwarteten Abstimmung. Der Rückzug auf eine minimale wechselseitige Verständigung bietet die Möglichkeit, diesen Mangel an gemeinsamen konventionenbasierten Stützen $\mathrm{zu}$ beheben. Jeder richtet sich in dieser minimalistischen Weise nach den Handlungen des anderen, ohne dass die Unsicherheit hinsichtlich des erwarteten Engagements in der jeweiligen Situation beseitigt wird. So liefert etwa der Arzt bei einer Konsultation dem Patienten die minimalen konventionenbasierten Grundlagen einer Intersubjektivität (Heath); ${ }^{66}$ wie er jedoch den Patienten $»$ rahmt ${ }^{67}$ behält er streng für sich oder teilt es anderen nur hinter verschlossener Tür mit. Der Patient dagegen kann dem Arzt lediglich lückenhafte Fragmente seiner eigenen Rahmen liefern. Die Koordination vollzieht sich hier über einen gemeinsamen Zugriff auf Fragmente von Handlungsregimes, die weitgehend getrennt voneinander bleiben. ${ }^{68}$ Die Koexistenz von Handlungsregimes in eigentlich strikt separaten oder separat gehaltenen Territorien stellt somit die auf die Spitze getriebene topographische Konstellation dar, in der sich das Auffinden konventionenbasierter Stützen erübrigt. Eine Pragmatik der Konventionen muss darauf bedacht sein, diese territorialen Trennungen der Koordinationsformen zu vermeiden; sie muss im Gegenteil versuchen, mittels soziologischer Erhebungen das MiteinanderVerkehren der Handlungsregimes in der Hoffnung zu aktivieren, die möglichen konventionenbasierten Grundlagen für Begegnungen zwischen solchen Personen zu verstärken, die ansonsten in teilweise voneinander abgeschotteten Welten handeln.

\section{Die Worte und die Handlungen: Die Prüfung der Pragmatik}

51 In methodologischer Hinsicht verknüpft eine soziologische Pragmatik zwei mögliche Perspektiven auf das Handeln. Die erste besteht darin, die im Ruhezustand verharrenden, anhand von Objekten, Schriften oder - ganz allgemein - Spuren menschlicher Tätigkeit materialisierten konventionenbasierten Stützen zu betrachten. Die zweite Perspektive besteht in der Beobachtung der konkreten Handlungen. Diese zweite Perspektive ergänzt die erste insofern, als sie einen Zugang $\mathrm{zu}$ all jenen Ressourcen vermittelt, die nur in belebter Form bestehen und nur über menschliche Handlungen aktualisiert werden (Gesten, Sprechakte), die ihrerseits wieder mit der Funktionsweise der Objekte und der Existenz von lebenden Wesen verknüpft sind. Mit ihr lassen sich sämtliche Vermittlungsprozesse darstellen, die über die Handlungen von Personen die Existenz von Handlungsregimes in konkreten Situationen gewährleisten. Diesbezüglich ist von Bedeutung, was uns die Ethnomethodologen zu sagen haben. Noch das kleinste Handlungssegment erweitert die Skala der möglichen Vermittler, die - wie man feststellt - unerlässlich sind, um den Formen der Koordination Gestalt zu verleihen, und bietet die Möglichkeit, allein schon angesichts der unbeweglichen 
Stützen unvermutete Vermittler zu entdecken. In einer Soziologie des common sense kann man, wie in der Sprachphilosophie, auf fiktive oder persönlich erlebte Beispiele zurückgreifen, indem man beim Leser die Kompetenzen abruft, die man mit ihm teilt. Um die lokalen Formen der Koordination ausfindig zu machen, muss man sich auf das Terrain begeben, auf dem sie empirisch fassbar sind. ${ }^{69}$ Der zweite Zugang zum Handeln - die Beobachtung der Personen im Handlungsvollzug - bietet im Übrigen die Möglichkeit, zwischen "aktiven«, in konkreten Situationen verwendeten und "passiven« Bezugspunkten zu unterscheiden, die in den im Ruhezustand befindlichen Stützen vorkommen, in den konkreten Handlungen jedoch nur eine sehr untergeordnete Rolle spielen. ${ }^{70}$

Die soziologische Pragmatik weist somit einen doppelten Bezug zu den Diskursen der Personen auf. Einerseits stattet sie die zentralen Begriffe, nämlich diejenigen, die ihre Zielrichtungen und ihre pragmatische Dichte bestimmen, mit Formen der Koordination aus. Wir haben gesehen, dass bestimmte Begriffe es den Personen ermöglichen, die mit den spezifizierten Formen der Koordination verbundenen Erwartungshorizonte zu umreißen. In der Rhetorik erfüllen diese Begriffe die Funktion von Fluchtpunkten (»Liebe«, "Gerechtigkeit", "Schönheit«, "Vergnügen«, in spezialisierten Welten aber auch »Klinik«, »Persönlichkeitsschutz«, "Behandlungsvertrag«, usw.). ${ }^{71}$ Aufgrund dieser Funktion ist ihre Position gegenüber den Handlungen nicht eindeutig. Nach Perelman sind sie gerade aufgrund ihrer Allgemeingültigkeit, ja sogar ihrer Mehrdeutigkeit als "verschwommene Begriffe « in der Lage, Ideale zu umreißen. ${ }^{72}$ Durch die Autonomisierung des Diskurses laufen sie jedoch Gefahr, ihre referentielle Funktion einzubüßen. Eine Pragmatik zeigt auf, wie sich diese Ressourcenkomplexe entfalten, die für die sich auf diese Begriffe beziehenden Handlungen notwendig sind. Diese Komplexe verbinden die Sprache mit dem Körper, den Objekten und der Umgebung. Die soziologische Pragmatik nähert sich hier einer Pragmatik der Sprache an, da sie, wie bei Wittgenstein, sämtliche konkreten Situationen zum Einsatz bringen will, in denen Worte gebraucht werden. Hier geht es jedoch um eine erweiterte Pragmatik, die sich, über die Worte hinaus, mit dem Erkennen bunt zusammengesetzter, wechselseitig verknüpfter Ressourcenkomplexe befasst.

$\mathrm{Zu}$ den Diskursen der Personen unterhält die soziologische Pragmatik ein anderes, kritischeres Verhältnis. Sie betont die Diskrepanzen zwischen Worten und Handlungen. Sie zeigt all die Vermittlungsleistungen auf, die von den Personen zur Aktivierung der ruhenden Dispositive erbracht werden. Und dadurch zeigt sie auch, was in der damit einhergehenden Rhetorik nicht erscheint. Die Ethnographie der wissenschaftlichen Tätigkeiten etwa macht sämtliche lokalen, zur Konstruktion wissenschaftlicher Tatsachen notwendigen Vorgänge sichtbar, die aber in die von den Wissenschaftlern selbst erstellten Forschungsberichte keinen Eingang finden. Die Soziologie zeigt hier, worin das Handeln über den Diskurs der Personen hinausgeht. Durch den Nachweis des Vorhandenseins ausschließlich passiver Bezugspunkte verweist sie zugleich darauf, inwieweit beim konkreten Handeln bestimmte Bezugspunkte unberücksichtigt bleiben. Das gilt etwa für die Regeln, von denen man annimmt, dass sie dieses Handeln anleiten. Sie sorgen für eine gewisse Wachsamkeit, was den Realismus der mit Worten gleichgesetzten Formen des Handelns anbelangt: Bestimmte Worte verfügen über keinerlei pragmatische Bedeutungserweiterung oder haben diese verloren.

Man wird nun besser verstehen, dass eine pragmatische Orientierung nicht vom Weg einer soziologischen Kritik wegführt, wenn sie das epistemologische Primat des Kalküls 
aufgibt, sondern diesen lediglich verlegt. In einer Theorie des - bewussten oder unbewussten - Kalküls ist die Kritik bereits in dem von Beginn an verwendeten Akteursmodell vorhanden. Sie bezieht ihre treibende Kraft nicht nur aus einem auf die Handelnden gerichteten Verdacht, sondern auch aus der Tatsache, dass dieser Verdacht durch die erhobenen empirischen Daten nur untermauert werden kann. Die Sprache des Kalküls ist das Bild, mit dem im Kopf der Soziologe die Erhebung beginnt und wieder beendet. Die kritische Energie bleibt mit allem, was sie an Scharfblick, Desillusionierung, Entzauberung, Anprangerung und moralischer Wachsamkeit mit sich bringt, konstant darauf gerichtet, das Kalkül offenzulegen. Die soziologische Pragmatik stellt einen anderen Bezug zum Diskurs der Personen und ihren Handlungen her. Ihr geht es darum, die Dichte der Worte aufzuzeigen und gleichzeitig zu untersuchen, in welchem Maß diese Worte den Personen tatsächlich als aktive Bezugspunkte dienen. Sie nimmt gewissermaßen »die Personen beim Wort« und unterzieht die Diskurse einer pragmatischen Prüfung. Die dabei geübte Kritik ist nicht Teil des Ausgangsmodells, sondern ist eines der möglichen Ergebnisse der Untersuchung.

\section{BIBLIOGRAPHIE}

Akrich, M. (1993): »Les objets techniques et leurs utilisateurs«, in: Conein, B. / Dodier, N. / Thévenot, L. (Hg.): Les objets dans l'action. De la maison au laboratoire, Paris, Edition der EHESS (Raisons pratiques, 4), S. 35-57.

Austin, J. L. (2007 [1962]): Zur Theorie der Sprechakte. Deutsche Bearbeitung von Eike von Savigny, Stuttgart, Reclam.

Barthélémy, M. (1990): „Voir et dire l'action. La normalisation des comportements des jeunes autistes«, in: Pharo, P. / Quéré, L. (Hg.): Les formes de l'action. Sémantique et sociologie, Paris, Edition der EHESS (Raisons pratiques, 1), S. 195-226.

Baszanger, I. (1991): »Déchiffrer la douleur chronique. Deux figures de la pragmatique médicale«, Sciences sociales et santé, IX (2), S. 31-78.

Baszanger, I. (1992): »Les chantiers d'un interactionniste«, Einleitung zu Strauss, A.: La trame de la négociation, Paris, L'Harmattan, S. 11-64.

Becker, H. (1982): Art worlds, Berkeley u.a., University of California Press.

Bessy, C. (1991): Licenciements économiques, formes de gestion de la main d'ouvre et dynamiques de l'emploi, Dossiers du Centre d'Etudes de l'Emploi, Nr. 38.

Bessy, C. / Chateauraynaud, F. (1993): „Le savoir-prendre. Enquête sur l'estimation des objets«, Techniques et culture, 20, S. 105-134.

Boltanski, L. (1990): L'amour et la justice comme compétences. Trois essais de sociologie en action, Paris, Métailié.

Boltanski, L / Thévenot, L. (2007 [1991]): Über die Rechtfertigung. Eine Soziologie der kritischen Urteilskraft. Aus dem Französischen von Andreas Pfeuffer, Hamburg, Hamburger Edition. 
Callon, M. (Hg.) (1989): La science et ses réseaux, Paris, La Découverte.

Chateauraynaud, F. (1991): La faute professionelle. Une sociologie des conflits de responsabilité, Paris, Métailié.

Conein, B. (Hg.) (1986): Lexique et faits sociaux, Lille, Presses Universitaires de Lille,(Lexique, 5).

Conein, B. (1990): »La cuisine dans tous ses états: cognition située et coordination de l'action«, Réseaux, 43, S. 99-110.

Conein, B. / Jacopin, E. (1993): »La planification dans l'action, les objets dans l'espace«, in: Conein, B. / Dodier, N. / Thévenot, L. (Hg.): Les objets dans l'action. De la maison au laboratoire, Paris, Editions der EHESS (Raisons pratiques, 4), S. 59-84.

Desrosières, A. (2005 [1993]): Die Politik der großen Zahlen. Eine Geschichte der statistischen Denkweise. Aus dem Französischen von Manfred Stern, Berlin / Heidelberg, Springer.

Dodier, N. (1993): L'expertise médicale. Essai de sociologie sur l'exercice du jugement, Paris, Métailié. Eymard-Duvernay, F. / Marchal, E. (1993): S'accorder avec les usagers. A l'interface d'un office HLM et de ses locataires, Paris, Centre d'Etudes de l'Emploi, rapport pour le commissariat au Plan.

Foucault, M. (1973 [1963]): Die Geburt der Klinik. Eine Archäologie des ärztlichen Blicks. Aus dem Französischen von Walter Seitter, München, Hanser.

Fujimura, J. H. (1992): „Crafting science: Standardized packages, boundary objects and stranslation «", in: Pickering, A. (Hg.): Science as Practice and Culture, Chicago, University of Chicago Press, S. 168-211.

Gadamer, H.-G. (1960): Wahrheit und Methode. Grundzüge einer philosophischen Hermeneutik, Tübingen, Mohr.

Garfinkel, H. (1967): Studies in ethnomethodology, Englewood Clidffs, Prentice Hall.

Goffman, E. (1977 [1974]): Rahmenanalyse. Ein Versuch über die Organisation von Alltagserfahrungen. Übersetzt von Hermann Vetter, Frankfurt a.M., Suhrkamp.

Gomart, E. (1993): Esquisse d'un modèle pour comprendre la consultation du médecin homéopathe, Mémoire de DEA (Diplomarbeit), EHESS, Paris.

Habermas, J. (1981): Theorie des kommunikativen Handelns, 2 Bde., Frankfurt a.M., Suhrkamp

Heath, C. (1986): Body movement and speech in medical interaction, Cambridge, Cambridge University Press.

Knorr-Cetina, K. (1984): Die Fabrikation von Erkenntnis. Zur Anthropologie der Naturwissenschaft, Frankfurt a.M., Suhrkamp.

Koselleck, R. (1979): Vergangene Zukunft. Zur Semantik geschichtlicher Zeiten, Frankfurt a.M., Suhrkamp.

Lafaye, C. (1990): „Situation tendues et sens ordinaires de la justice au sein d'une administration municipale«, Revue française de sociologie, 31, S. 199-223.

Latour, B. (1989): La science en action, Paris, La Découverte.

Latour, B. (1995 [1991]): Wir sind nie modern gewesen. Versuch einer symmetrischen Anthropologie. Aus dem Französischen von Gustav Roßler, Berlin, Akademie-Verlag.

Latour, B. / Woolgar, S. (1988 [1979]): La vie de laboratoire, Paris, La Découverte (engl. Original von 1979). 
Leenhardt, M. (1984 [1947]): Do Kamo. Die Person und der Mythos in der melanesischen Welt. Übersetzt von Eva Brückner-Pfaffenberger, Frankfurt a.M. u.a., Ullstein Verlag.

Leroi-Gourhan, A. (1980 [1964]): Hand und Wort. Die Evolution von Technik, Sprache und Kunst. Übersetzt von Michael Bischoff, Frankfurt a.M., Suhrkamp.

Licoppe, C. (1993): Dans le jardin secret des scientifiques: les ethnographies de laboratoire, Entwurf, Université de Paris VII.

Lynch, M. (1985): Art and artefact in laboratory science: A study of shop work and shop talk in a research laboratory, London, Routledge and Kegan Paul.

Mol, A. (1995): »La topographie comme méthode d'investigation du savoir. De la coexistence de diverses anémies«, in: Culture technique, 25-26, S. 285-305 (Original in Niederländisch).

Mol, A. / Law, J. (1994): »Regions, networks and fluids: Anaemia and social topology«, Social Studies of Science, 24, S. 641-671.

Norman, D. A. (1988): The psychology of everyday things, New York, Basic Books.

Norman, D. A. (1993): »Artefacts cognitifs«, in: Conein, B. / Dodier, N. / Thévenot, L. (Hg.) (1993): Les objets dans l'action. De la maison au laboratoire, Paris, Edition der EHESS (Raisons pratiques, 4), S. 15-34.

Perelman, C. (1989): Rhetoriques, Brüssel, Editions de l'Université de Bruxelles.

Pollak, M. (1988 [1990]): Die Grenzen des Sagbaren. Lebensgeschichte von KZ-Überlebenden als Augenzeugenberichte und als Identitätsarbeit. Aus dem Französischen von Hella Beister, Frankfurt a.M. / New York, Campus.

Poulet, G. (1952): Etudes sur le temps humain, 4 Bde., Paris, Plon.

Quéré, L. (1989): „L'argument sociologique de Garfinkel«, Réseaux, 27, Dezember.

Rawls, J. (1975 [1971]): Eine Theorie der Gerechtigkeit. Aus dem Amerikanischen von Hermann Vetter, Frankfurt a.M., Suhrkamp.

Ricœur, P. (1996): Das Selbst als ein Anderer. Aus dem Französischen von Jean Greisch, München, Fink.

Sacks, H. / Schegloff, E. A. (1973): »Opening up closings«, Semiotica, 7, S. 289-327.

Sahlins, M. (1986 [1981]): Der Tod des Kapitän Cook. Geschichte als Metapher und Mythos als Wirklichkeit in der Frühgeschichte des Königreichs Hawaii. Aus dem Amerikanischen von Hans Medick u. Michael Schmidt, Berlin, Wagenbach.

Schütz, A. (1987): Le chercheur et le quotidien, Paris, Méridiens-Klingensieck (= Auszug aus: Schütz, A. (1971-1972): Gesammelte Aufsätze, 3 Bde., Den Haag, Nijhoff).

Silverman, D. (1987): Communication and Medical Practice. Social Relations in the Clinic, London, Sage Publications.

Star, S. L. / Griesemer, J. R. (1989): »Institutional ecology, stranslation « and boundary objects: Amateurs and professionals in Berkeley's Museum of Vertebrate Zoology«, Social Studies of Science, 19, S. 387-420.

Strauss, A. L. (1982): „Social Worlds and legitimation processes«, in: Denzin, N. (Hg.): Studies in Symbolic Interaction, Bd. 4, London / Greenwich, JAI Press, S. 171-190.

Suchman, L. (1987): Plans and Situated Actions. The Problem of Human-Machine Communication, Cambridge, Cambridge University Press. 
Thévenot, L. (1989): „Equilibre et rationalité dans un univers complexe«, Revue économique, Sonderausgabe Economie des conventions, 40 (2), S. 147-198.

Thévenot, L. (1990): "L'action qui convient«, in: Pharo, P. / Quéré, L. (Hg.): Les formes de l'action. Sémantique et sociologie, Paris, Edition der EHESS (Raisons pratiques, 1), S. 39-70.

Walzer, M. (2006 [1983]): Sphären der Gerechtigkeit. Ein Plädoyer für Pluralität und Gleichheit. Aus dem Englischen von Hanne Herkommer, Frankfurt a.M. / New York, Campus.

Wittgenstein, L. (1980 [1961]): Philosophische Untersuchungen, 2. Aufl., Frankfurt a.M., Suhrkamp.

\section{NOTES}

1. Habermas (1981), Rawls (1975 [1971]).

2. Garfinkel (1967).

3. Ricœur (1996).

4. Walzer (2006 [1983]).

5. Boltanski / Thévenot (2007 [1991]).

6. Strauss (1982), Becker (1982), Star / Griesemer (1989), Fujimura (1992).

7. Schütz (1987).

8. Wir verweisen an dieser Stelle auf die bemerkenswerte Einführung Baszangers (1992) zu den Arbeiten von Strauss, in denen die Zusammenhänge zwischen Interaktionismus und Pragmatismus aufgezeigt werden.

9. Siehe dazu etwa Knorr-Cetina (1984), Lynch (1985), Latour / Woolgar (1988 [1979]) sowie die zu diesem Thema vorgestellte kritische Bilanz Licoppes (1993).

10. Suchman (1987), Norman (1988), Conein (1990).

11. Suchman (1987).

12. Conein / Jacopin (1993).

13. Dodier (1993).

14. Norman (1993).

15. Leroi-Gourhan (1980 [1964]).

16. Bessy / Chateauraynaud (1993).

17. Akrich (1993).

18. Leroi-Gourhan (1980 [1964]).

19. Zur Definition der Begriffe "Erfahrung der Vergangenheit« und "Erwartungshorizont«, in deren Verbindung das Verhältnis der Individuen zurzeit und insbesondere zur historischen Zeit zum Ausdruck kommt, verweisen wir auf Koselleck (1979).

20. Poulet (1952).

21. Conein / Jacopin. (1993).

22. Boltanski / Thevenot (2007 [1991]).

23. Leroi-Gourhan (1980 [1964]), Norman (1993).

24. Latour (1989).

25. Boltanski / Thevenot (2007 [1991]).

26. Quéré (1984).

27. Conein (1986).

28. Sacks / Schlegloff (1973), Conein (1986).

29. Garfinkel (1967), Barthélémy (1990).

30. Suchman (1987).

31. Suchman (1987).

32. Boltanski / Thevenot (2007 [1991]). 
33. Gadamer (1960).

34. Dodier (1993).

35. Latour (1989), Akrich (1993).

36. Wittgenstein (1980 [1961]).

37. Goffman (1977 [1974]).

38. Austin (2007 [1992]).

39. Silverman (1987).

40. Baszanger (1991).

41. Dodier (1993). Bessy und Chateauraynaud (1993) zeigen in ihrer Studie zur Bewertung von Objekten, dass die Personen in zwei Regimes involviert sind: in das Regime der »Macht der Objekte«, wobei die Personen deren »Zauber unterliegen«, und, über die Zwischenstufe des "Regimes des Verstehens« in das Regime der »Objektivierung" dieser Objekte, wobei sich die Personen von ihren Emotionen leiten lassen, um in den Traditionen ein Verfahren zur Bewertung des Objekts zu finden. »Die beschriebenen Situationen zeigen, dass es nicht einerseits Laien gibt, die einer Beeinflussung unterliegen, und auf der anderen Seite die professionellen Inhaber von objektivierungsinstrumenten, über die sie das Monopol haben. Professionelle wie Laien bewegen sich gleichzeitig zwischen verschiedenen Regimes, und eine gelungene Einigung beruht genau darauf, dass sie die zum Ausdruck gebrachten Empfindungen und Beurteilungen über die Art und Weise, wie sie das Objekt fassen, in Übereinstimmung bringen können« (a.a.O., S.21; Übersetzung: Jörg Ohnacker).

42. Boltanski (1990).

43. Leenhardt (1984 [1947]).

44. Akrich (1993) zeigt etwa, wie die Gestalter eines technischen Objekts (in diesem Fall ein Zusatzgerät, um bestimmte Video-Abonnementdienstleistungen über einen Fernseher abzurufen) unter großen Schwierigkeiten versuchen, das objekt an seine voraussichtliche häusliche Umgebung anzupassen (Verbindung zu anderen Objekten, Anordnung der Teile, Konzentration mehrerer Funktionen im selben Objekt).

45. Lafaye (1990).

46. Boltanski / Thévenot (2007 [1991]).

47. Silverman (1987), Goffman (1977 [1974]), Dodier (1993).

48. Callon (1989), Latour (1989).

49. Zur »Komplexität« der Welt in Bezug auf Gerechtigkeit vgl. Thévenot (1989).

50. Goffman (1977 [1974]), Dodier (1993).

51. Pollak (1988 [1990]).

52. Chateauraynaud (1991).

53. Boltanski / Thévenot (2007 [1991]), Boltanski (1990).

54. Boltanski / Thévenot (2007 [1991]), Eymard-Duvernay / Marchal (1993).

55. Desrosières (2005 [1993]).

56. Boltanski (1990).

57. Latour (1995 [1991]).

58. Star / Griesemer (1989).

59. Dodier (1993).

60. Bessy (1991).

61. Mol (1995).

62. Mol / Law (1994) gehen von drei Formen des Raums aus: Regionen, Netzwerke, fließende Räume.

63. Boltanski (1990).

64. Thévenot (1990).

65. Foucault (1973 [1963]), Baszanger (1991), Dodier (1993).

66. Heath (1986). 
67. Dodier (1993).

68. Das Problem, das sich stellt, wenn Personen aufeinandertreffen, denen nur sehr wenige konventionsspezifische Grundlagen gemeinsam sind, zeigt sich ganz deutlich, wenn vorher voneinander abgeschottete lokale Kulturen Beziehungen zueinander aufnehmen. Sahlins (1986 [1981]) konnte in seinen Untersuchungen die tragischen Missverständnisse aufzeigen, die den Begegnungen zwischen James Cook und den Bewohnern von Hawaii zugrunde lagen.

69. Dodier (1993).

70. Gomart (1993).

71. Perelman (1989).

72. Perelman (1989), S. 127.

\section{INDEX}

Schlüsselwörter : Handlungstheorie, Konventionen, Koordinationsformen, Räumlichkeit, Zeitlichkeit

Mots-clés : conventions, distribution dans l'espace, formes de coordination, temporalité, théorie de l'action

\section{AUTEURS}

\section{NICOLAS DODIER}

Nicolas Dodier ist Soziologe, Forschungsdirektor am »Institut National de la Santé et de la Recherche Médical« (INSERM) sowie Studiendirektor an den Écoles des Hautes Études en Sciences Sociales (EHESS). Nähere Informationen finden Sie hier. 